\title{
TALK TEST: A SIMPLE ALTERNATIVE TO IDENTIFY LACTATE THRESHOLDS DURING PROGRESSIVE CYCLING EXERCISE
}

\author{
Leonardo De Lucca ${ }^{1}$, Fernando Roberto de Oliveira² ${ }^{2}$, Carl Foster ${ }^{3}$, \\ and Lorival José Carminatti ${ }^{4}$ \\ ${ }^{1}$ University of Southern Santa Catarina - UNISUL, Brazil \\ ${ }^{2}$ Federal University of Lavras, Brazil \\ ${ }^{3}$ University of Wisconsin-La Crosse, USA \\ ${ }^{4}$ University of Santa Catarina State, Brazil
}

Original scientific paper

DOI 10.26582/k.53.1.3

\begin{abstract}
:
The assessment of speech production difficulty has been used to control aerobic exercise intensity through the Talk Test method. The aim of this study was to compare the Talk Test variables to lactate thresholds using an incremental test. Thirteen male subjects performed an incremental cycle test to identify the first lactate threshold (LT1), second lactate threshold (LT2) and transition points of the Talk Test. During the incremental exercise test subjects read aloud a standard paragraph at the end of each stage. The last stage at which the subjects could talk comfortably, the first stage at which the subjects could not talk comfortably, and the first stage at which they definitely could not talk comfortably were referred to as the last positive (LP), equivocal (EQ) and negative stages (NEG), respectively. The power output ( $88 \pm 22 \mathrm{~W})$ and heart rate at the first lactate threshold $(115 \pm 15 \mathrm{bpm})$ were significantly lower $(\mathrm{p}<.05)$ than the power output at the last positive $(127 \pm 26 \mathrm{~W})$ and heart rate $(131 \pm 21 \mathrm{bpm})$ at the last positive. The power output and heart rate at the second lactate threshold $(169.5 \pm 22.6 \mathrm{~W} ; 157 \pm 14 \mathrm{bpm})$ were not significantly different $(\mathrm{p}=.195)$ from power output and heart rate at the equivocal $(178.8 \pm 30.3 \mathrm{~W} ; 161 \pm 15 \mathrm{bpm})$. The heart rate at the second lactate threshold and equivocal were significantly correlated $(r=.57 ; \mathrm{p}<.05)$. In conclusion, this study showed that the equivocal stage of the Talk Test can be used as a simple tool to indirectly identify the second lactate threshold in healthy men during cycling exercise.
\end{abstract}

Keys words: exercise test, speech production, physiological thresholds

\section{Introduction}

The lactate threshold (LT) represents the onset of a metabolic acidosis during incremental exercise testing (IET) and correlates well with endurance performance (Sjodin \& Jacobs, 1981). Some authors have proposed methods for noninvasively determining LT, based on heart rate responses during an IET (Conconi, et al., 1982), ventilatory threshold (VT) (Ahmaidi, et al., 1993) and rating of perceived exertion (RPE) (Moreira, et al., 2010). VT and LT determination requires expensive equipment, and complex or invasive laboratory procedures. These limitations decrease access to exercise testing. More simple and less expensive tools related to exercise intensity assessment might be useful to predict these metabolic thresholds. Another method advocated for prescribing exercise training intensity is based on the Talk Test method (TT) or the ability of an individual to maintain a conversation during exercise (Dehart-Beverley, Foster, Porcari, Fater, \& Mikat, 2000; Foster, et al., 2008, 2018; Gillespie, McCormick, Mermier, \& Gibson, 2015; Persinger, Foster, Fater, \& Porcari, 2004; Quinn \& Coons, 2011; Recalde, et al., 2002; RodríguezMarroyo, Villa, García-Lopez, \& Foster, 2013; Schroeder, Foster, Porcari, \& Mikat, 2018). The rationale for this method is based on the premise that exercising at or above the VT generally does not allow complete conversational sentences without pausing for breaths and thus serves well as a means of estimating the ceiling training intensity (DehartBeverley, et al., 2000).

The main advantage of the TT method is its simplicity. It suggests that if the exercise intensity is sufficient so that the patient can "just respond to conversation", then the exercise intensity is likely to be within accepted ranges of exercise training intensity (Foster, et al., 2018). In recent years, the 
validity of this simple guideline has been systematically evaluated. The ability to comfortably converse during exercise (i.e., to pass the Talk Test) has been shown to produce exercise intensities consistently within the parameters suggested in clinical guidelines for exercise training in a variety of populations including healthy individuals (Recalde, et al., 2002), clinically stable patients with cardiovascular disease (Voelker, et al., 2002), and athletes (Gillespie, et al., 2015; Rodríguez-Marroyo, et al., 2013).

Previous studies have consistently indicated that both the exercise intensity at the VT and the first intensity stage at which a subject cannot unequivocally comfortably speak occur at approximately the same point. (Persinger, et al., 2004; Recalde, et al., 2002). In addition, Quinn and Coons (2011) indicated that all of the physiological and perceptual responses that were recorded at the equivocal and negative stages of the TT (including $\mathrm{VO} 2$, heart rate, RPE, minute ventilation, \%VEmax, \%VO2max, and \%HRmax) were either slightly or significantly higher than those same responses recorded at either the lactate or ventilatory thresholds.

Although a great deal is known about the relationship between TT, LT and VT, little is known about what lactate and ventilatory turn points are consistently estimated by these TT stages. Previous research has demonstrated the occurrence of at least two distinct turn points in the lactate and ventilation curves during an IET (Ahmaidi, et al., 1993). The first abrupt increase in blood lactate concentration (BL) was widely used as a gold standard for noninvasive threshold determinations by ventilation or gas exchange parameters (Yoshida et al., 1987). For example, a log-log transformation of blood lactate versus $\mathrm{VO}_{2}$ (Beaver, et al., 1985) is an accepted way for the detection of this 'first lactate turn point', which may approximate BL levels around 2 mmol $\cdot \mathrm{l}^{-1}$ (Kindermann, et al., 1979). With increasing workload above the first LT the subject reaches the second turn point at which lactate production equals maximal lactate clearance capacity. This has been called the maximal lactate steady state (MLSS) (Heck, et al., 1985) or 'onset of blood lactate accumulation' (Sjodin \& Jacobs, 1981). It reflects the workload beyond which continuous exercise will not lead to a steady state and is used as one of the high limit intensities during endurance training. This 'second lactate turn point' was shown to correlate well with MLSS (Fell, 2008), approximates the BL level of 4 mmol-1-1 in some subjects (Heck, et al., 1985; Mader, Liesen, Heck, Philippi, \& Rost, 1976) and is also defined as the 'individual anaerobic threshold' which possibly better reflects the individual lactate level at the MLSS (Ahmaidi, et al., 1993; Stegmann \& Kindermann, 1981).

The aims of this study were to measure physiological variables at both the first (LT1) and second lactate threshold (LT2) and to determine if TT outcomes (LP, EQ and NEG) elicit physiolog- ical variables that were comparable to those seen at these lactate based thresholds. In addition, we wished to compare these Talk Test outcomes with relative upper and lower exercise intensity ranges, corresponding with the ACSM recommendations (ACSM, 2011) for moderate-to-vigorous intensities according to heart rate exercise prescriptions. We hypothesized that: (1) The LT1 physiological variables would be not significantly different than the LP stage of TT; (2) The LT2 variables would not be significantly different from those measured at the EQ stage of TT; (3) The NEG variables would be significantly greater than LT2 variables; (4) The EQ stage variables would represent the ACSM ceiling exercise prescription range ( $\left.\leq 90 \% \mathrm{HR}_{\max }\right)$; and (5) The LP would define a lower exercise intensity range $\left(60-85 \% \mathrm{HR}_{\max }\right)$.

\section{Methods}

\section{Experimental approach to the problem}

To test whether TT can be used to estimate the LT1 and LT2, 13 healthy adults performed an incremental test. It included measurement of blood lactate concentrations to determine the LT1, LT2 and HR at the LT1, LT2 and the TT stages. Additionally, during this test, the subjects read out aloud a standard paragraph at the end of each exercise stage and reported their ability to speak comfortably. The last stage at which the subjects could definitely speak comfortably (LP), the first stage at which the subjects could not unequivocally speak comfortably (EQ), or the first stage where they definitely could not speak comfortably (NEG) was compared with the LT1 and LT2.

\section{Subjects}

Thirteen healthy nonsmoking male volunteers participated in the study. They were all familiar with the procedure of maximal exercise testing and either performed some kind of regular exercise on a noncompetitive level (less than $5 \mathrm{~h}$ of sport training a week), or were sub-elite college athletes (futsal or handball). Their characteristics are shown in Table 1 . The subjects were familiar with the exer-

Table 1. Participants' characteristics and exercise test results (Mean $\pm S D)$

\begin{tabular}{lc}
\hline Variables & Mean $\pm \mathrm{SD}$ \\
\hline Age (years) & $20.6 \pm 2.0$ \\
Body mass $(\mathrm{kg})$ & $75.9 \pm 14.0$ \\
Body height $(\mathrm{cm})$ & $176.8 \pm 7.4$ \\
Maximal workload $(\mathrm{W})$ & $230 \pm 23$ \\
Maximal workload $\left(\mathrm{W} \cdot \mathrm{kg}^{-1}\right)$ & $3.09 \pm 0.54$ \\
HRmax $(\mathrm{bpm})$ & $187 \pm 9$ \\
Peak [La] $\left(\left.\mathrm{mmol} \cdot\right|^{-1}\right)$ & $11.7 \pm 2.7$ \\
Peak RPE & $9 \pm 1$ \\
\hline
\end{tabular}


cise testing protocol as all had previously performed exercise tests in our laboratory. The subjects were instructed to avoid strenuous training sessions 48 hours before the testing sessions, and caffeine ingestion 12 hours before testing. No food intake was allowed three hours before testing. All participants provided written informed consent, and the protocol was approved by the Institutional Review Board of Santa Catarina State University.

\section{Procedures}

Following anthropometric data collection, an incremental exercise test was performed on an electronically braked bicycle ergometer (Ergoline 900, Blitz, Germany) in the upright position. The exercise protocol consisted of a 5-minute warm-up at $50 \mathrm{~W}$, a 3-minute initial workload at $50 \mathrm{~W}$ followed by stepwise increases of $25 \mathrm{~W}$ every 3-min until the maximal tolerable load was achieved. The tests were performed between 14:00 p.m. and 18:00 p.m. in the laboratory with the room temperature set for $22^{\circ} \mathrm{C}$. During testing, a Polar S610I HR monitor (Polar Electro, Kempele, Finland) was fitted around the subject's chest and used to measure participants' HR each 10-s before the end of each stage. Maximal HR was recorded as the highest value obtained for the last 10 -second before exhaustion. Immediately after each 3-minute stage blood was withdrawn for lactate determination. Capillary blood samples $(25 \mathrm{uL})$ were taken from a previously hyperemized earlobe to measure blood lactate concentration. Blood lactate was analyzed in duplicate using a lactate analyzer (YSI 1500 Sport Lactate Analyzer, Yellow Springs, Inc., Yellow Springs, OH) that had been calibrated according to the manufacturer's recommendations. During the last 10 seconds of each exercise stage, the rating of perceived exertion (RPE) was recorded using the category ratio (0-10) RPE scale (Borg, 1998). The subjects from this study had earlier performed incremental tests in our laboratory using the RPE scale and were familiar with it. The end criteria was in accordance with the traditional physiological standards (Howley, et al., 1995): (1) elevated blood lactate concentration ( $\geq 8$ $\mathrm{mmol} / \mathrm{L}) ;(2)$ elevated HR ( $\geq 90 \%$ of ( $220-$ age) and (3) near maximal RPE (>8).The maximal workload was determined as the highest workload subjects could maintain for a complete stage plus the interpolated workload from the following incomplete stages (Kuipers, et al., 1985). Verbal encouragement was given to ensure maximum physical effort.

After the test, the data were plotted on graphs [la] vs. workload, the first lactate threshold (LT1) was identified as the lowest value of the [la] / load equivalent according to Berg et al., (1990). The second lactate threshold (LT2) was identified by the Dmax ADAPT. This method is proposed by the Australian Institute of Sport (Bourdon, 2000). It consists in calculating the greatest distance between the curve formed by the points of the [la] as a function of the workload and a straight line, which is drawn from the LT1 and the last point of the curve. The point which represents the greatest distance between the curve and the straight line was considered as the LT2. The modified D-max lactate threshold has been shown to provide a valid measure of power output and heart rate that can be maintained during endurance cycling exercise in veteran cyclists (Fell, 2008).

In TT the subjects recited aloud a standard paragraph (38 words) from a Brazilian classic birthday song during the last 30 seconds of each exercise stage. The subjects were familiar with this paragraph. A cue card was located in front of the subject to allow reference to the text if necessary. Immediately after reciting the paragraph, the participant was asked: "Can you talk comfortably?" Only three possible answers were recorded: "Yes", which was referred to as a "positive" result; "perhaps", which was referred to as an "equivocal" result; and "no", which was referred to as a "negative" result. The last exercise stage at which the participants were still unequivocally able to talk comfortably was referred to as the last positive stage (LP). The first exercise stages at which the participants were not entirely certain about their ability to talk comfortably were referred to as the equivocal stage (EQ). Finally, the first stage at which the cyclist could not talk comfortably was referred to as the negative stage (NEG).

\section{Statistical analysis}

Statistical analysis was carried out using the statistical package SPSS Version 21.0 (IBM Corp., Armonk, NY, USA). The Gaussian distribution for the data was verified by the Shapiro-Wilk goodnessof-fit test ( $\mathrm{Z}$ value $<1.0)$. Heart rate $(\mathrm{HR})$, work load (WL), \%HRmax and \% WLmax were the variables analyzed in each technique. The absolute and relative WL values at EQ, LP, NEG, LT1 and LT2 were compared using Friedman's nonparametric test and the Wilcoxon's post-hoc test. The Spearman Rank correlation was used to verify the degree of association between these variables. The absolute and relative HR values for LP, EQ, NEG, LT1 and LT2 were tested with ANOVA and Bonferroni post-hoc measurements. The degree of association between these values was verified by Pearson's correlation, considering $\mathrm{p}<.05$.

\section{Results}

The power output at LP was significantly higher than at LT1 and significantly lower than at LT2, EQ and NEG. There were no significant differences between the HR and \%HRmax corresponding to LT2 and EQ ( $p=.476)$. The HR and \%HRmax at LT1 were significantly lower than at LP. There were 
Table 2. Physiological variables at the lactate thresholds and Talk Test stages (Mean $\pm S D$ )

\begin{tabular}{lcccc}
\hline & Workload $(\mathbf{W})$ & HR $(\mathbf{b p m})$ & \%maximal workload & \%HRmax \\
\hline LT1 & $88.4 \pm 21.9^{\mathrm{a}}$ & $115 \pm 15^{\mathrm{a}}$ & $38.3 \pm 8.6^{\mathrm{a}}$ & $61.5 \pm 7.4^{\mathrm{a}}$ \\
LT2 & $169.5 \pm 22.6^{\mathrm{b}}$ & $157 \pm 14^{\mathrm{b}}$ & $73.5 \pm 5.4^{\mathrm{b}}$ & $83.7 \pm 6.7^{\mathrm{b}}$ \\
LP & $126.9 \pm 25.9$ & $131 \pm 21$ & $55.0 \pm 8.3$ & $70.0 \pm 9.1$ \\
EQ & $178.8 \pm 30.3^{\mathrm{b}, \mathrm{c}}$ & $161 \pm 15^{\mathrm{b}, \mathrm{c}}$ & $77.6 \pm 8.4^{\mathrm{b}, \mathrm{c}}$ & $86.3 \pm 7.3^{\mathrm{b}, \mathrm{c}}$ \\
NEG & $203.4 \pm 29.3^{\mathrm{a}, \mathrm{c}}$ & $174 \pm 13^{\mathrm{a}, \mathrm{c}}$ & $88.4 \pm 8.2^{\mathrm{a}, \mathrm{c}}$ & $93.1 \pm 5.5^{\mathrm{a}, \mathrm{c}}$ \\
\hline
\end{tabular}

Note. LT1 - first lactate threshold; LT2 - second lactate threshold; LP - last positive stage of the Talk Test; EQ - last equivocal stage of the Talk Test; NEG - first negative stage of the Talk Test. a significantly different from LT2, LP and EQ ( $p<.05)$. ${ }^{b}$ significantly different from LP $(p<.05) .{ }^{c}$ significantly different from LT1 $(p<.05)$.

Table 3. Correlation analysis of workload and HR at LT1, LT2, LP, EQ and NEG

\begin{tabular}{lcccccccc}
\hline & \multicolumn{3}{c}{ Workload $(\rho$ Spearman) } & \multicolumn{3}{c}{ HR (r Pearson) } \\
\cline { 2 - 10 } & LT2 & LP & EQ & NEG & LT2 & LP & EQ & NEG \\
\hline LT1 & $0.72^{* *}$ & $0.57^{*}$ & 0.45 & 0.45 & $0.73^{* *}$ & $0.78^{* *}$ & $0.61^{*}$ & 0.54 \\
LT2 & & $0.64^{*}$ & 0.53 & 0.53 & & $0.64^{*}$ & $0.57^{*}$ & 0.35 \\
LP & & & $0.74^{* *}$ & $0.74^{* *}$ & & & $0.88^{* *}$ & $0.85^{* *}$ \\
EQ & & & & $0.99^{* *}$ & & & & $0.87^{* *}$ \\
\hline
\end{tabular}

Note. LT1 - first lactate threshold; LT2 - second lactate threshold; LP - last positive stage of the Talk Test; EQ - last equivocal stage of the Talk Test; NEG - first negative stage of the Talk Test. ${ }^{* *} p<.01 ;{ }^{*} p<.05$

no significant differences between the power output at the LT2 (WLLT2) and the power output at EQ (WEQ) ( $\mathrm{p}=.195)$ (Table 2). The power output at NEG was significantly higher than at LT1, LT2, LP and EQ $(p<.001)$. There were no significant differences between relative workload (\%WLmax) at LT2 and EQ ( $\mathrm{p}=.283)$.

Non-significant, but representative correlations between workload at LT2 and EQ ( $\mathrm{r}=.53 ; \mathrm{p}=.061)$ were observed (Table 3). Significant correlations between the PO at LT2 and LP were observed (Table 3). Significant correlations between HR at LT2 and EQ were observed. The PO and HR at LT1 and LP were correlated. Significant correlations between \%HRmax at LT1 and LP were observed $(r=.70 ; p<.01)$.

\section{Discussion and conclusions}

The main findings of this study were that the LT1 (whether expressed as either power output or HR) was generally overestimated by the LP stage of the TT, and that the EQ stage of the TT was a reasonable approximation of the LT2. The present study determined that the TT method might be an alternative for analyzing the metabolic thresholds during an incremental cycling test to exhaustion in sub-elite college athletes, although the TT appeared incapable of resolving the LT1. We also found that TT outcomes might be used to prescribe the relative upper exercise intensity range, which corresponded with the ACSM recommendations for vigorous intensities according to HR exercise prescriptions (ACSM, 2011). Specifically, while the
EQ stage of the TT may be useful for determining the LT2 and the upper limit for exercise recommendation, the LP stage of the TT may not be appropriate for the minimum exercise intensity range, at least when estimated by blood lactate diagnostic criteria. Although previous studies have shown that the TT may indicate intensities and physiological parameters similar to VT markers in cyclists (Gillespie, et al., 2015), runners (Quinn \& Coons, 2011), healthy people (Persinger, et al., 2004), students (Dehart-Beverley, et al., 2000), and people with illness (Voelker, et al., 2002), to our knowledge, this study suggests that the TT responses may not be adequate to define the LT1. The closer relationship between the VT and TT markers makes sense in that the main ventilatory requirement of the TT is to suppress breathing frequency, at exactly the point where both breathing frequency and total ventilation would normally be increasing (Foster, et al., 2018). Other authors (Creemers, et al., 2018) have shown that the end tidal [CO2], a marker of arterial [CO2], increases significantly during the $\mathrm{TT}$, especially above the VT where the VCO2 is normally increasing sharply, thus the correspondence between the VT and TT appears to be linked mechanistically. However, Schroeder et al. (2018) have shown that relatively short ( $\sim 30$ words) speech provoking paragraphs, such as used in this study (38 words), tend to overestimate the power output at the VT1. Thus, the present finding that the LP stage of the TT overestimated the LT1 is congruent with other findings which used VT as the first metabolic threshold. 
In this study, we showed that HR, \%HRmax, $\mathrm{PO}$ and \% POmax at EQ could be used for indicating LT2. In light of these findings, it seems reasonable to modify our approach to TT use, whereas it may have broad implications for the demarcation of the domains of exercise intensity in humans. These findings can be likened to those of Persinger et al. (2004) who demonstrated that $\mathrm{VO}_{2}$ values at $\mathrm{VT}$ were not significantly different from $\mathrm{VO} 2$ at LP and EQ. In another study with young adults, Recalde et al. (2002) found no significant differences between $\mathrm{VO} 2, \% \mathrm{VO}_{2 \text { peak }}$, $\mathrm{HR}$ and $\% \mathrm{HR}_{\max }$ at VT and EQ with a significant correlation between these variables. Voelker et al. (2002) found no significant difference between $\mathrm{VO} 2$ at VT, LP and EQ as well as correlation between $\mathrm{VO} 2$ at VT and VO2 at LP, EQ and NEG in patients with heart disease. Similar to the findings of the present study, these researchers found significant differences for all outcomes between VT and NEG as well as VT and LP. In these investigations, the VT was defined using the V-slope method, one of the most frequently used methods for the determination of the first threshold (Beaver, et al., 1985). If it is accepted that the first curvilinear rise in VE is called the "(first) ventilatory threshold (VT1), that could be the first "aerobic" threshold, thus VT is a noninvasive determination of the first lactate threshold or the called LT1 in our study.

Although we have not found significant differences in workload at LT2 and EQ, we found significant differences between LP and LT1. However, Quinn and Coons (2011) demonstrated that HR and VO2 measured at the VT significantly under-represented values measured at LP, EQ and NEG and that data from the LT more closely represented the LP measurements. The authors used 3-min stages treadmill running protocol and showed that all the values measured at the LT were significantly higher than those recorded at the VT. These findings have led us to reconsider the validity of LP for determining the LT1. Futhermore, a correlation was seen between the PO, \%POmax HR and \%HRmax at LT1 and LP (Table 3). The interest in comparing LT1 variables with the TT values was made necessary by the absence of comparative analyzes that also addressed the first transition threshold (LT1 or VT1) in the literature. Moreover, the terminology used to differentiate the first and second transition thresholds leads to controversy about the interpretation of the results. None of these studies clarified what is called the 'VT', so we adopted the concept that the curve of minute ventilation (VE) showed a curvilinear slope pattern with two break points. The first coincided with the 'aerobic threshold' (VT1 or LT1), the second with the 'anaerobic threshold' (VT2 or LT2) (James, et al., 1989). Most of the studies were carried out in patients with chronic disease, which would be more likely to present an abnormal ventilatory response and the early rise in lactic acidosis during incremental exercise test (Tumminello, et al., 2007). Furthermore, because of rapidly developing dyspnea, chronic disease patients may neither show the first (Midorikawa, et al., 1997) nor the second LT (Beaver, et al., 1985). Other patients may exhibit elevated blood lactate levels at rest or with minimal exercise (Haller, et al., 1989) associated with exaggerated ventilatory response (Haller, et al., 1989; Patessio, et al., 1993). Decreased oxidative capacity of the affected mitochondria causes shortening of the aerobic-anaerobic transition making discrimination of the first from the second threshold difficult (Haller, et al., 1989).

In the present study, it can be shown that the EQ stages are close to LT2 and above LT1, and that LP, contrary to our previous hypothesis, does not correspond to intensities close to LT1. Rodriguez-Marroyo et al. (2013) showed that no significant differences were found between VT and EQ and values at LP were significantly less than those obtained at VT and EQ. They also found no significant differences between the physiological parameters (HR and RPE) at the respiratory compensation threshold (RCT) and NEG. However, these parameters were significantly different than those at EQ. These authors used two ventilatory turn points as we did in our study with lactate. According to some studies (Binder, et al., 2008) VT is equivalent to LT1 and RCT to LT2, thus our study indicates that NEG occurs in intensities above the LT2 when lactate measures are used to identify physiological thresholds.

Dehart-Beverley et al. (2000) demonstrated that when individuals were no longer able to maintain a comfortable conversation during exercise, the intensity would be around $90 \%$ of HRmax and would serve as an upper limit to exercise prescription. These researchers reported that at EQ the subjects were exercising on average at $87.8 \%$ of HRmax. Persinger et al. (2004) showed values of $89 \%$ in treadmill and $82 \%$ in the cycle ergometer. These values are very close to those found in the present study, where subjects were on average of $86.3 \%$ of HRmax at EQ. In addition, NEG derived variables indicate the perception of inability to maintain comfortable speech and would be above the upper limit for $90 \%$ aerobic exercise of HRmax (American, 2011) corroborating with the results of other studies (Dehart-Beverley, et al., 2000; Persinger, et al., 2004). The $\% \mathrm{HR}$ max found (93.1\%) is similar to that reported by Persinger et al. (2004), who found values of $91.6 \%$ of the HRmax in the cycle ergometer, and Volker et al. (2002), who showed 94.8\% in patients with heart disease exercising on treadmill. Therefore, we can further strengthen the premise that TT is a good method to delimit exercise intensity using HR values according to the range of $55-90 \%$ of HRmax established by ACSM 
(American, 2011) as an aerobic exercise training zone.

However, the study had some technical limitations. First, the protocol with 3-minute stages became necessary, since between 3-5 $\mathrm{min}$ it is possible to reach steady states of HR and VO2. Therefore, the TT variables were limited by 3-minute intervals and might have affected the temporal resolution of the relation with other variables; the stages of difficulty in speech could be more accurate with a greater variability of power values and better analyzed with the physiological variables if the protocol had allowed a greater number of stages. Furthermore, for the TT determination we used a paragraph that contained 38 words and 126 letters in total, characterizing a long and probably longer passage that would be commonly spoken during the exercise. This method is similar to that of Persinger et al. (2004) and Recalde et al. (2002) who adopted a passage with 31 words and different from that used by Dehart-Beverly et al. (2000) who used 101 words. However, Shroeder et al. (2018) have recently shown that short speech passages (31 words) tend to cause TT results to overestimate the power output at VT and RCT, probably because the time for end tidal $\mathrm{CO} 2$ to increase is inadequate (Creemers, et al., 2017). Longer passages (62 and 93 words) produce a much smaller average error in estimating the PO at both VT and RCT. This presents a dilemma in that shorter speech passages are easier to use in laboratory evaluations, and that spontaneous speech during exercise is likely to use shorter phrasing units. Furthermore, because it was a subjective method, where the subject determined the degree of speech difficulty, the actual situation of vocalization impairment due to ventilatory parameters may not have been well elucidated and could be evaluated less subjectively by other evaluators. Another point that should be questioned is whether the subjects recited the paragraph aloud, because if the tone of voice was low, the subjects could exercise at a higher intensity, since the increase in ventilatory frequency, which occurs at VT, interfere with the ventilatory control that is necessary for vocalization.

Moreover, some physiological variables have their responses altered during exercise due to speech production. It was reported that $\mathrm{VO} 2$ was reduced by speech production during three exercise intensities due to a reduction in ventilation to maintain an adequate expiratory flow for phonation. Speech production also caused a significant increase in [La] during the lowest intensity, but did not change this variable in the other two higher intensities (Meckel, et al., 2002). According to these authors, this increase in [La] may indicate a greater use of anaerobic sources of energy production by speech production.

In conclusion, our results show that when participants could speak comfortably (LP), they were exercising below their LT2 and above LT1, whereas at the EQ stages of the TT participants were consistently approaching LT2. It was hypothesized that the power output and HR values recorded at the LP stage of the TT would be close to those same measurements recorded at the LT1. Our results were not in agreement with this hypothesis. It was also hypothesized that the same measures recorded at the NEG stage of the Talk Test would be significantly higher than those same measures recorded at the LT2. Results were in agreement with this hypothesis. Participants were exercising within pre-established exercise intensity guidelines during the last point (LP) at which they could comfortably respond to conversation (55\% Wmax and $70 \%$ HRmax). However, when participants could no longer respond comfortably to conversation during exercise, they were either outside of ACSM exercise intensity guidelines at NEG (93.1\% HRmax) or were exercising at the upper end of these guidelines (86.3\% HRmax) at EQ.

Taken together, the present results and other evidence from the literature suggest that the suppression of breathing frequency required by speaking may take a reasonably long time to have a physiological effect, particularly at low exercise intensities (e.g. LP TT or LT1). Thus, relatively longer speech passages ( $>60$ words) may be required to allow the TT to be a meaningful surrogate of low intensity physiologic thresholds. At higher rates of relative energy expenditure (e.g. LP EQ or LT2), the rate of accumulation of arterial $\mathrm{CO} 2$ appears to be adequate to cause matching of the TT stage to the higher physiological thresholds (VT2, LT2), even with the minor disruption of breathing frequency caused by short speech-provoking passages.

\section{Practical applications}

According to the results presented, the TT seems to be a reasonable method to estimate the LT2 and is valid enough to control aerobic exercise intensity in a simple way and without additional costs. The EQ seems to occur at intensities close to LT2 and below 90\% HRmax. Coaches and practitioners who prescribe physical exercise to improve health-related physical fitness may require athletes and students to have a comfortable conversation at intensities between LT1 and LT2 (light and moderate exercise), converse with some discomfort (EQ) at intensities at or near the LT2 (moderate to heavy exercise) and very uncomfortable conversation or speech impossibility (NEG) to reach intensities above the LT2 (heavy exercise). Using the TT during training sessions, events can provide a practical and convenient method to help monitor an athlete's exercise intensity with respect to LT1 and LT2 and thus provide practical estimations of the physiological domains (light, moderate or heavy exercise). 


\section{References}

Ahmaidi, S., Hardy, J., Varray, A., Collomp, K., Mercier, J., \& Prefaut, C. (1993). Respiratory gas exchange indices used to detect the blood lactate accumulation threshold during an incremental exercise test in young athletes. European Journal of Applied Physiology and Occupational Physiology, 66, 31-36.

American-AMCS (American College of Sport Medicine). (2011). Quantity and quality of exercise for developing and maintaining cardiorespiratory, musculoskeletal, and neuromotor fitness in apparently healthy adults: Guidance for prescribing exercise. Medicine and Science in Sports and Exercise, 43(7), 1334-1359.

Beaver, W., Wasserman, K., \& Whipp, B. (1985). Improved detection of the lactate threshold during exercise using a log-log transformation. Journal of Applied Physiology, 59, 1936-1940.

Berg, A., Jakob, E., Lehmann, M., Dickhuth, H. H., Huber, G., \& Keul, J. (1990). Current aspects of modern ergometry. Pneumologie, 44(1), 2-13.

Binder, R. K., Wonisch, M., Corra, U., Cohen-Solal, A., Vanhees, L., Saner, H., \& Schmid, J. P. (2008). Methodological approach to the first and second lactate threshold in incremental cardiopulmonary exercise testing. European Journal of Preventive Cardiology, 15(6), 726-734. https://doi.org/10.1097/HJR.0b013e328304fed4.

Borg, G. (1998). Borg's Perceived Exertion and Pain Scales. Human Kinetics Publishers.

Bourdon, P. (2000). Blood lactate transition thresholds: concepts and controversies. In C. Gore (Ed.), Physiological tests for elite athletes (pp. 55-65). Human Kinetics.

Conconi, F., Ferrrari, M., Ziglio, P., Droghetti, P., \& Codeca, L. (1982). Determination of the anaerobic threshold by a noninvasive field test in runners. Journal of Applied Physiology, 52, 862-873.

Creemers, N., Foster, C., Porcari, J. P., Cress, M. L., \& De Koning, J. J. (2018). The physiological mechanism behind the talk test. Kinesiology, 49(1), 3-8. https://doi.org/10.26582/k.49.1.15

Dehart-Beverley, M., Foster, C., Porcari, J. P., Fater, W., \& Mikat, R. P. (2000). Relationship between the talk test and VentilatoryThreshold. Clinical Exercise Physiology, 1(2), 34-38.

Fell, J. (2008). The modified D-max is a valid lactate threshold measurement in veteran cyclists. Journal of Science and Medicine in Sport, 11, 460-463.

Foster, C., Porcari, J. P., Anderson, J., Paulson, M., Smaczny, D., Webber, H., Doberstein, S. T., \& Udermann, B. (2008). The Talk Test as a Marker of Exercise Training Intensity. Journal of Cardiopulmonary Rehabilitation and Prevention, 28(1), 24-30.

Foster, C., Porcari, J. P., Foster, C., Porcari, J. P., Ault, S., Doro, K., Dubiel, J., Engen, M., Kolman, D., \& Xiong, S. (2018). Exercise Prescription When There Is No Exercise Test : the Talk Test. Kinesiology, 50(1), 1-16.

Gillespie, B. D., McCormick, J. J., Mermier, C. M., \& Gibson, A. L. (2015). Talk Test as a practical method to estimate exercise intensity in highly trained competitive cyclists. Journal of Strength an Conditioning Research, 29(4), 894-898.

Haller, R., Lewis, S., Estabrook, R., DiMauro, S., Servidei, S., \& Foster, D. (1989). Exercise intolerance, lactic acidosis, and abnormal cardiopulmonary regulation in exercise associated with adult skeletal muscle cytochrome coxidase deficiency. Journal of Clinical Investigation, 84, 155-161.

Heck, H., Mader, A., Hess, G., Mucke, S., Muller, R., \& Hollmann, W. (1985). Justification of the 4-mmol/1 lactate threshold. International Journal of Sports Medicine, 6, 117-130.

Howley, E., Bassett, D., \& Welch, H. (1995). Criteria for maximal oxygen uptake: review and commentary. Medicine and Science in Sports and Exercise, 27, 1292-1301.

James, N., Adams, G., \& Wilson, A. (1989). Determination of anaerobic threshold by respiratory frequency. International Journal of Sports Medicine, 10, 192-196.

Kindermann, W., Simon, G., \& Keul, J. (1979). The significance of the aerobic-anaerobic transition for the determination of work load intensities during endurance training. European Journal of Applied Physiology and Occupational Physiology, 42, 25-34.

Kuipers, H., Verstappen, F., Keizer, H., Geurten, P., \& Van Kranenburg, G. (1985). Variability of aerobic performance in the laboratory and its physiological correlates. International Journal of Sports Medicine, 6, 197-201.

Mader, A., Liesen, H., Heck, H., Philippi, H., Rost, R., Schuerch, P., \& Hollmann, W. (1976). Zur Beurteilung der sportartspezifischen Ausdauerleistungsfa” higkeit im Labor. Sportarzt Und Sportmedizin, 27, 80-112.

Meckel, Y., Rotstein, A., \& Inbar, O. (2002). The effects of speech production on physiologic responses during submaximal exercise. Medicine and Science in Sports and Exercise, 34(8), 1337-1343.

Midorikawa, J., Hida, W., Taguchi, O., Okabe, S., Kurosawa, H., \& Mizusawa, A. (1997). Lack of ventilatory threshold in patients with chronic obstructive pulmonary disease. Respiration, 64, 76-80.

Moreira, S., Oliveira, R., Nakamura, F., \& Campbell, C. (2010). Lactate threshold prediction by blood glucose and rating of perceived exertion in people with type 2 diabetes. Perceptual and Motor Skills, 111(2), 365-378.

Patessio, A., Casaburi, R., Carone, M., Appendini, L., Donner, C., \& Wasserman, K. (1993). Comparison of gas exchange, lactate, and lactic acidosis thresholds in patients with chronic obstructive pulmonary disease. $A m$ Rev Respir Dis, 148, 622-626.

Persinger, R., Foster, C., Fater, D. C., \& Porcari, J. P. (2004). Consistency of the Talk Test for Exercise Prescription. Medicine and Science in Sports and Exercise., 36(9), 1632-1636. https://doi.org/10.1249/01.MSS.0000074670.03001.98 
Quinn, T. J., \& Coons, B. A. (2011). The talk test and its relationship with the ventilatory and lactate thresholds. Journal of Sports Sciences, 29(11), 1175-1182. https://doi.org/10.1080/02640414.2011.585165

Recalde, P. T., Foster, C., Skemp-Arlt, K. M., Fater, D. C. W., Neese, C. A., Dodge, C., \& Porcari, J. P. (2002). The "Talk Test" as a simple marker of Ventilatory Threshold. South African Journal of Sports Medicine, 9, 5-8.

Rodríguez-Marroyo, J. A., Villa, J. G., García-Lopez, J., \& Foster, C. (2013). Relationship between the Talk Test and Ventilatory Threshold in well-trained cyclists. Journal of Strength and Conditioning Research, 27(7), $1942-1949$.

Schroeder, M. M., Foster, C., Porcari, J. P., \& Mikat, R. P. (2018). Effects of speech passage length on accuracy of predicting metabolic thresholds using the talk test. Kinesiology, 49(1), 9-14. https://doi.org/10.26582/k.49.1.14

Sjodin, B., \& Jacobs, I. (1981). Onset of blood lactate accumulation and marathon running performance. International Journal of Sports Medicine, 2, 23-26.

Stegmann, H., \& Kindermann, W. (1981). Lactate kinetics and individual anaerobic threshold. International Journal of Sports Medicine, 2, 160-165.

Tumminello, G., Guazzi, M., Lancellott, P., \& Pierard, L. (2007). Exercise ventilation inefficiency in heart failure: pathophysiological and clinical significance. European Heart Journal, 28, 673-677.

Voelker, S., Foster, C., Porcari, J. P., Skemp, K., Brice, G., \& Backes, R. (2002). Relationship Between the Talk Test and Ventilatory Threshold in Cardiac Patients. Clinical Exercise Physiology, 4(2), 120-123.

Yoshida, T., Chida, M., Ichioka, M., \& Suda, Y. (1987). Blood lactate parameters related to aerobic capacity and endurance performance. European Journal of Applied Physiology and Occupational Physiology, 56, 7-11.

Submitted: June 21, 2019

Accepted: May 7, 2020

Published Online First: March 5, 2021

Correspondence to:

Leonardo De Lucca, M.Sc.

University of Southern Santa Catarina - UNISUL

Research Group in Sport Management and Physical

Activity

Palhoça, SC - Brazil

Phone: +55 48996128252

Email: leonardo.lucca@unisul.br

\section{Acknowledgments}

The equipment was provided by the University of Santa Catarina State, Center of Health and Sports Sciences. We sincerely thank all subjects who kindly devoted their time and energy to testing. This research was made possible by the University of Santa Catarina State and their commitment to students' education. Our gratitude is extended to the team of the laboratory of Human Performance for their generosity in allowing us to conduct this study and utilize the laboratory resources. 